Artigo de Revisáo/Atualizaçáo

\title{
Saúde mental de estudantes de terapia ocupacional: revisão de escopo
}

\section{Mental health of occupational therapy students: scoping review}

\author{
Teresinha Cid Constantinidis $^{\mathrm{a}}$ (D), Thelma Simóes Matsukura ${ }^{\mathrm{b}}$ (D) \\ ${ }^{a}$ Universidade Federal do Espírito Santo - UFES, Vitória, ES, Brasil \\ bUniversidade Federal de São Carlos - UFSCar, São Carlos, SP, Brasil
}

Como citar: Constantinidis, T. C., \& Matsukura, T. S. (2021). Saúde mental de estudantes de terapia ocupacional: revisão de escopo. Cadernos Brasileiros de Terapia Ocupacional, 29, e2139.

https://doi.org/10.1590/2526-8910.ctoAR2139

\begin{abstract}
Resumo
Este estudo focaliza a saúde mental de estudantes de graduação de terapia ocupacional por meio de revisão de escopo, norteada pela pergunta: Como a literatura científica trata as questóes relacionadas à saúde mental de estudantes de terapia ocupacional? Utilizou-se o guia de revisão de escopo Preferred Reporting Items for Systematic reviews and Meta-Analyses extension for Scoping Reviews (PRISMA-ScR) Checklist. Foram incluídos estudos empíricos e teóricos, publicados em inglês, francês, espanhol ou português, sem recorte temporal determinado. O levantamento dos estudos foi realizado nas bases de dados Scopus, Redalyc, Google Acadêmico, na Biblioteca Digital de Teses e Dissertaçôes (BDTD), resultando na análise de 15 estudos. A análise de conteúdo dos artigos delineou núcleos de sentido que indicaram a realização de atividades práticas e a imersão do aluno no campo de estágio como fontes de estresse e sofrimento psíquico. Além disso, estudos apontam as estudantes de terapia ocupacional como mais afetadas em sua saúde mental, se comparadas a estudantes de outras áreas da saúde. Ressalta-se a importância de investigação das causas subjacentes ao sofrimento psíquico deste estudante, além da necessidade de se considerar variáveis, como gênero e situação socioeconômica. Considera-se que o estudo contribuirá para o planejamento de estratégias de promoção de saúde mental, prevençáo de sofrimento psíquico e cuidado com esses estudantes, além de medidas que visem manter este aluno na universidade e concluir seu curso com qualidade.
\end{abstract}

Palavras-chave: Estudantes, Saúde Mental, Sofrimento Psíquico, Terapia Ocupacional.

\section{$\underline{\text { Abstract }}$}

This study focuses on the mental health of undergraduate occupational therapy students through a scoping review, guided by the question: How does the scientific literature deal with issues related to the mental health of occupational therapy 
students? The Preferred Reporting Items for Systematic reviews and Meta-Analyzes extension for Scoping Reviews (PRISMA-ScR) Checklist was used. Empirical and theoretical studies, published in English, French, Spanish, or Portuguese were included, with no determined time frame. The survey of the studies was carried out in the databases Scopus, Redalyc, Google Scholar, in the Digital Library of Theses and Dissertations, resulting in the analysis of 15 studies. The content analysis of the articles delineated nuclei of meaning that indicated the performance of practical activities and the immersion of the student in the internship field as sources of stress and psychological suffering. In addition, studies indicate that occupational therapy students are more affected in their mental health, compared to students from other areas of health. The importance of investigating the causes underlying this student's psychological distress is emphasized, in addition to the need to consider variables such as gender and socioeconomic situation. It is considered that the study will contribute to the planning of strategies for the promotion of mental health, prevention of psychological distress, and care for these students, in addition to measures aimed at keeping this student at the university and concluding his course with quality.

Keyword: Students, Mental Health, Mental Disorder, Occupational Therapy.

\section{Introduçáo}

Nos últimos anos, a saúde mental de estudantes universitários tem sido foco de preocupação social pela elevada prevalência de transtornos mentais entre este grupo de jovens (Orellana et al., 2020). Estudos recentes desenvolvidos no Brasil e no exterior afirmam que esta prevalência é maior se comparada a jovens não estudantes (Fórum Nacional de PróReitores de Assuntos Estudantis, 2016; Ibrahim et al., 2013; Leão et al., 2018). Pesquisa realizada sobre o perfil socioeconômico e cultural de estudantes de graduação das instituiçóes federais de ensino superior, realizada em 2018 (Fórum Nacional de Pró-Reitores de Assuntos Estudantis, 2019), aponta que o percentual de ideação de morte e pensamento suicida entre estudantes universitários brasileiros é de 10,8\% e 8,5\%, respectivamente; já na mesma pesquisa, realizada em 2014, o percentual de estudantes com ideação de morte era 6,1\%, enquanto pensamento suicida afetava 4\%. Desta forma, alertam que "Está acesa a luz vermelha da atenção à saúde mental” entre jovens universitários (Fórum Nacional de PróReitores de Assuntos Estudantis, 2019, p. 229).

Alguns estudos apontam que a adaptação à universidade pode não ser bem-sucedida, conduzindo a uma vulnerabilidade da saúde mental, trazendo sofrimento, emergindo conflitos de várias ordens, traços de transtornos mentais e, no extremo, processo suicida entre estudantes (Anversa et al., 2018; Carleto et al., 2018). Gonçalves \& Ambar (2015) apontam que existem sinais de violência e sofrimento psíquico, simbólicos e/ou físicos, entre estudantes que são ignorados pelas universidades. Os autores destacam que o sofrimento entre estudantes náo deixa de manifestar seus efeitos por meio de estresse, ansiedade, depressão, drogadição, suicídio, assim como altos índices de reprovação, trancamento e evasão escolar. Neste sentido, ressalta-se a importância de pesquisas sobre a natureza do sofrimento de estudantes para que respaldem a implementaçáo efetiva de açôes que promovam a saúde mental deste grupo de jovens, ou mesmo que previna tais transtornos. 
A maioria dos estudos nacionais e internacionais sobre a saúde mental de estudantes universitários é realizada com estudantes de medicina (Fond et al., 2019; Grace, 2018), mas mesmo tendo muitos pontos de intersecçáo com estudantes da área da saúde, o estudante de medicina não é modelo ilustrativo de outros cursos da área. Desta forma, verifica-se número crescente de estudos sobre a saúde mental de estudantes vinculados à pluralidade de cursos da área da saúde (Claudino et al., 2019; Kotera et al., 2019; Lima et al., 2019; Murakami et al., 2019)

Em relação aos estudos sobre saúde mental da estudante de terapia ocupacional, verifica-se que, na década de 1990, surgem as primeiras publicaçóes sobre o tema, centrando-se principalmente no em estresse ${ }^{1}$ e no sofrimento psicológico decorrentes das demandas do curso. Nos últimos anos, acompanhando o aumento de prevalência de transtornos mentais entre estudantes universitários, há um aumento na produção de estudos sobre a saúde mental da estudante de terapia ocupacional. Este estudo trata de uma revisão de escopo que visa analisar as contribuiçóes de pesquisas nacionais e internacionais sobre a saúde mental de estudantes de terapia ocupacional, além de identificar as tendências epistemológicas dos estudos sobre o tema e identificar os conceitos que subsidiam tais pesquisas.

\section{Método}

Trata-se de uma revisão de escopo que tem grande utilidade para sintetizar evidências de pesquisa e mapear a literatura existente em determinado campo em termos de sua natureza, recursos e volume (Peters et al., 2015). A presente revisão utiliza como referência o guia para relatório de revisão de escopo Preferred Reporting Items for Systematic reviews and Meta-Analyses extension for Scoping Reviews (PRISMA-ScR) Checklist (Tricco et al., 2018). Conforme os passos apontados pelo PRISMA-ScR, a pergunta norteadora da pesquisa foi elaborada tendo como elementos-chave a populaçáo ou participantes, conceitos e contexto. Assim, desenvolveu-se a pergunta: Como a literatura cientifica trata as questóes relacionadas à saúde mental de estudantes de terapia ocupacional? Nesta, participantes são estudantes universitários, o conceito é saúde mental destes estudantes e o contexto é o curso de terapia ocupacional.

Foram incluídos estudos empíricos e teóricos, publicados em inglês, francês, espanhol ou português, publicados até dezembro de 2019, dentre artigos, teses, dissertaçóes, monografias ou livros. Foram excluídos estudos sobre intervençóes, pois, entende-se que o tema central de tais investigaçóes está centrado em modelos, métodos e técnicas terapêuticas, tangenciando a questão desta revisão.

O levantamento dos estudos foi realizado entre os meses de março e maio de 2020, em três etapas. Na primeira etapa, a pesquisa foi realizada nas bases de dados Scopus e Redalyc. Na segunda etapa, foram examinadas as listas de referência dos artigos que surgiram na primeira etapa para garantir a utilização da literatura relevante do tema. $\mathrm{Na}$ terceira etapa, foram pesquisados os estudos não publicados no Google Acadêmico e na Biblioteca Digital de Teses e Dissertaçôes (BDTD). Em todas as etapas, foram utilizadas

\footnotetext{
${ }^{1}$ Dentre os estudos apresentados sobre o tema, neste artigo, e que apresentam algum referencial conceitual sobre estresse, consideram-no como uma síndrome de adaptação apresentada pelo indivíduo diante de presença de estímulo agressor, que pode desencadear desequilíbrio nas funçóes corporais, ocasionando doenças físicas e/ou emocionais (Murakami et al., 2019; Sanches et al., 2018).
} 
as palavras-chave: Saúde mental; sofrimento psíquico; transtorno mental; estudante de terapia ocupacional; aluno de terapia ocupacional para bases de dados nacionais e mental health; psychological distress; occupational therapy student; occupational therapy para bases internacionais, conforme apresentado na Tabela 1.

Tabela 1. Bases de dados, estratégia de busca e referências.

\begin{tabular}{|c|c|c|}
\hline Estratégia de busca & $\begin{array}{l}\text { Bases de dados/ } \\
\text { Recurso de } \\
\text { informaçáo } \\
\end{array}$ & $\begin{array}{l}\text { Referências } \\
\text { recuperadas }\end{array}$ \\
\hline $\begin{array}{c}\text { "Occupational Therapy Student" AND"Mental } \\
\text { Health"; "Occupational Therapy Student" AND } \\
\text { "Psychological Distress" }\end{array}$ & Scopus & 129 \\
\hline $\begin{array}{c}\text { "Saúde Mental" } A N D \text { "Estudante de Terapia } \\
\text { Ocupacional" } O R \text { "Aluno de Terapia Ocupacional"; }\end{array}$ & Redalyc & 07 \\
\hline $\begin{array}{l}\text { Sofrimento Psíquico } A N D \text { "Estudante de Terapia } \\
\text { Ocupacional" OR "Aluno de Terapia Ocupacional"; }\end{array}$ & Google Acadêmico & 230 \\
\hline Ocupacional" $O R$ "Aluno de Terapia Ocupacional"; & BDTD & 144 \\
\hline
\end{tabular}

Ao todo, foram encontrados 520 trabalhos, resultado da soma de 510 referências encontradas nas bases de dados e de 10 referências resultantes de análise de referencial de artigos selecionados. Foram excluídas 74 duplicações, resultando em 446 estudos para análise preliminar. Com base na leitura do título, foram excluídos 383 estudos que não atendiam ao critério de inclusão. Desta forma, foram selecionados 63 estudos restantes para leitura do resumo, dos quais foram excluídos 42 artigos por não atenderem ao critério de inclusão. Portanto, 21 artigos e duas dissertaçóes foram selecionados para leitura na íntegra. Destes, seis artigos foram excluídos, quatro deles por apresentarem temas relacionados à saúde mental, mas não correlacionarem com a saúde mental de estudantes de terapia ocupacional; um deles por apenas tangenciar o tema de saúde mental de estudantes na comparação de dois currículos específicos de cursos de terapia ocupacional de duas universidades; um deles por tratar de estudo de caso, mais voltado ao transtorno mental específico de estudante de terapia ocupacional sem relacionar com o curso. Deste processo, foram incluídos 15 estudos para a revisáo proposta. O processo de mapeamento dos dados é apresentado no fluxograma (Figura 1) elaborado com base no modelo do Prisma Flow Diagram.

Os 15 estudos foram exportados para o Mendeley ${ }^{\circ}$, gerenciador de referências que classifica os artigos segundo a autoria, título, ano de publicação e periódico. Os resultados das pesquisas foram tratados com base na Análise Temática de Conteúdo (Bardin, 2011). Após, as autoras fizeram a codificação dos dados por meio de leitura e busca de temas de forma independente. Posteriormente, foi realizada discussão sobre a codificação, entre as pesquisadoras, até chegar a um consenso no processo de categorização, propondo-se núcleos de sentido explicativos. 


\section{Resultados}

\section{Características dos estudos incluídos na revisáo}

As produçóes se concentram no período de 1993 a 2019. Na Figura 2, pode-se verificar uma constância maior de produçáo a partir de 2015, ou seja, nos últimos quatro anos. Além disso, observa-se que o número de produção aumentou nos últimos dois anos (Figura 2).

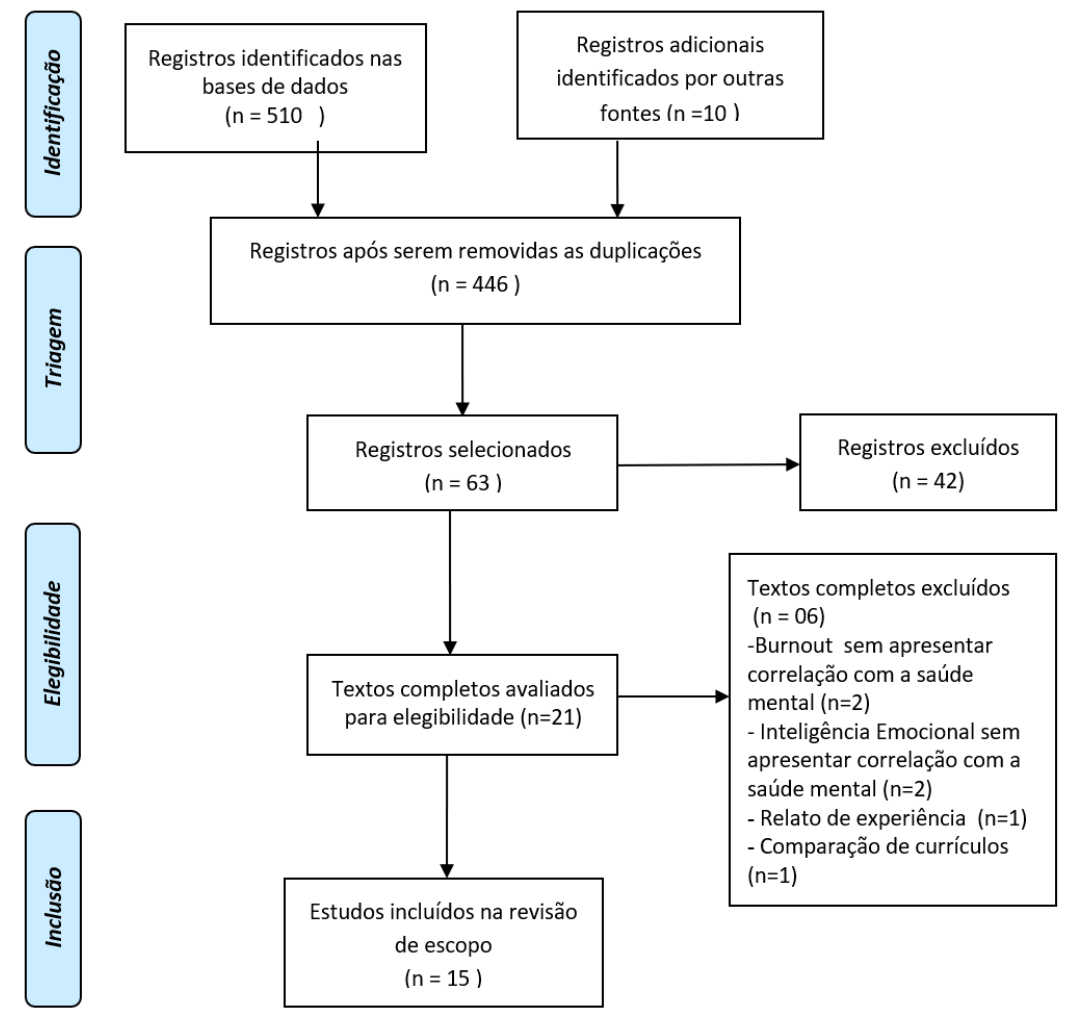

Figura 1. Fluxograma de busca. Fonte: Moher et al. (2009).

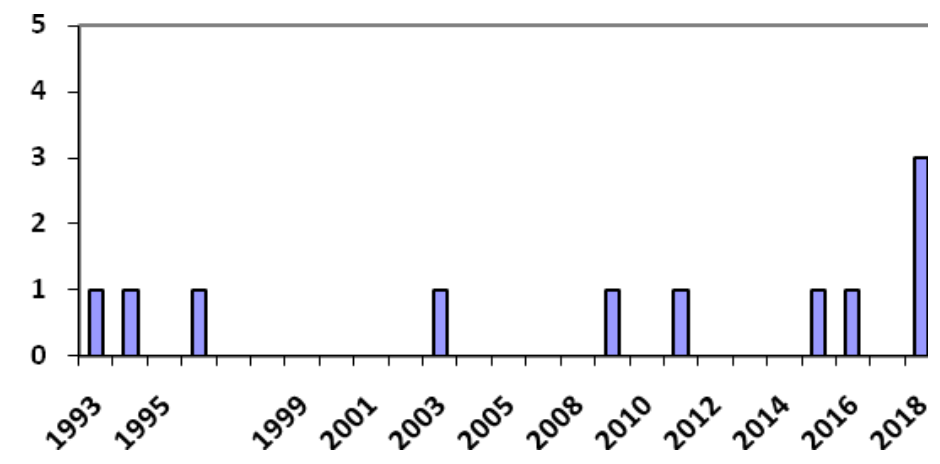

Figura 2. Número de Publicaçôes distribuído no intervalo de tempo. 
Em relação à distribuição geográfica das produções, seis estudos são nacionais (Arantes, 2018; Cavestro \& Rocha, 2006; Murakami et al., 2019; Pinho, 2018; Sanches et al., 2018), sendo quinze na totalidade. Países como Estados Unidos são representados por três trabalhos (McCombie, 2019; Mitchell \& Kampfe, 1993; Everly et al., 1994); Noruega é representada por dois trabalhos (Bonsaksen, 2015; Nerdrum et al., 2009); África do Sul também por dois trabalhos (Govender et al., 2015; Witt et al., 2019); Austrália (Gilbert \& Strong, 1997) e Reino Unido (Kotera et al., 2019) por um trabalho cada.

Os temas abordados, em relação à saúde mental de estudantes de terapia ocupacional, foram: estresse, transtorno mental, coping, autoestima, autocrítica, autocompaixão, autoeficácia, autoconfiança, resiliência e uso de álcool. Tais temas, em alguns estudos, aparecem combinados entre si e configuram, conforme Figura 3, a seguinte proporção:

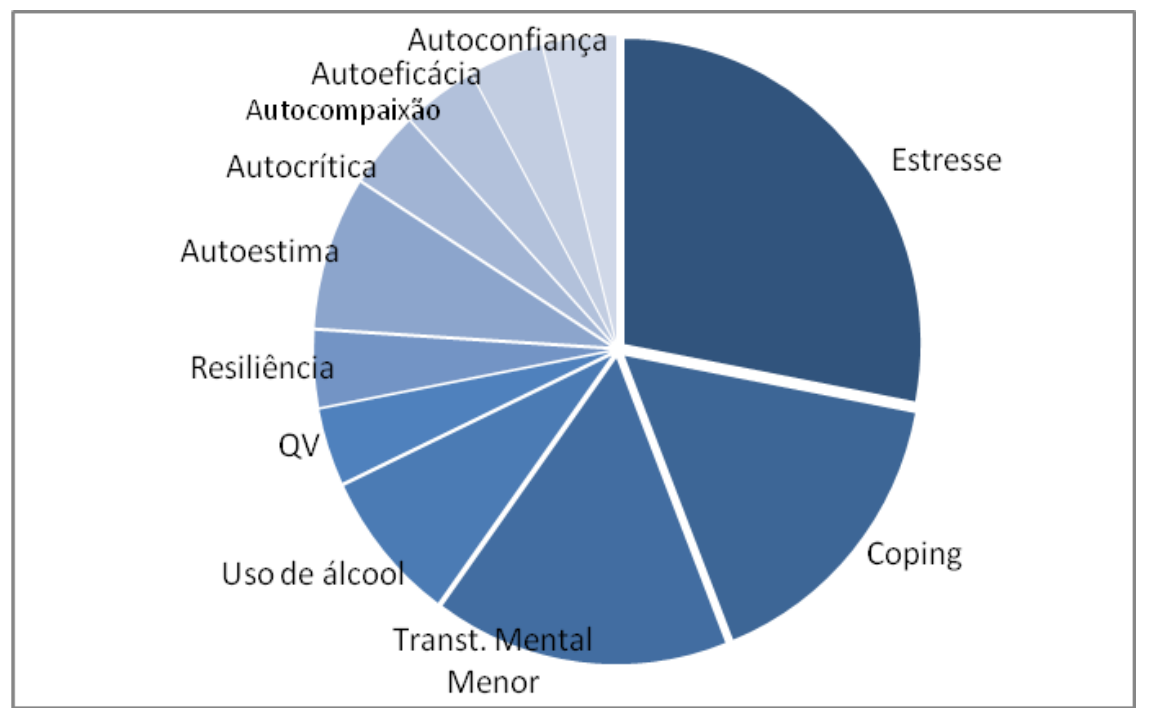

Figura 3. Variáveis distribuídas em proporção de estudos.

\section{Análise do conteúdo dos estudos}

Com base na análise do conteúdo dos artigos, foram encontradas regularidades que delinearam os seguintes núcleos de sentido explicativos: 1 . Saúde mental de estudantes de terapia ocupacional e especificidades do curso de graduação; 2. Saúde mental de estudantes de terapia ocupacional vista em paralelo à saúde mental de estudantes do campo da saúde; 3. Variáveis associadas à saúde mental de estudantes de terapia ocupacional.

\section{Saúde mental de estudantes de terapia ocupacional e especificidades do curso de graduaçáo}

O estágio curricular é citado, nestes artigos (Gilbert \& Strong, 1997; Govender et al., 2015; Mitchell \& Kampfe, 1993; Sanches et al., 2018; Everly et al., 1994; Witt et al., 2019), como principal fonte de estresse e ansiedade entre estudantes, 
podendo impactar a saúde mental desses jovens. Apesar de se tratar de estudos de diferentes países, como Brasil, África do Sul, Austrália e EUA, todos os currículos dos cursos de terapia ocupacional dos diferentes países, com variaçóes nas modalidades de formação, entre cursos de nível mestrado profissional ou bacharelado, todos oferecem estágio curricular nos últimos semestres, configurando-se como etapa fundamental de formação acadêmica do terapeuta ocupacional (World Federation of Occupational Therapists, 2016).

Desde as primeiras publicaçóes sobre o tema, a passagem para os estágios curriculares/profissionalizantes do curso de terapia ocupacional é vista como geradora de estresse nos estudantes. Mitchell \& Kampfe (1993) realizaram pesquisa em que avaliam as estratégias de enfrentamento de estresse e a percepção da experiência de passagem para o campo de estágio, entre estudantes do curso. Para tal fim, aplicaram o questionário Revised Way of coping checklist (WCCL) e questionário desenvolvido especialmente para o estudo, o Transition Questionnaire, a 104 estudantes de terapia ocupacional, dentre os quais estáo 88 mulheres e 12 homens, de 15 universidades dos Estados Unidos da América (EUA).

Assim, os autores verificaram que, diante do estresse, os estudantes se esforçam mais em estratégias positivas, como estratégia focada no problema e busca pelo suporte social, do que em estratégias negativas, como pensamentos negativos, de evasão e autopiedade. Em relação à percepção da transição, os resultados apontaram diferenças significativas entre os gêneros, sendo que as mulheres percebem o estresse mais fortemente que os homens. Os autores entendem que esta diferença é resultado de um número de variáveis, como estado civil, finanças, realocação de moradia (se o estágio é realizado em cidade diferente) etc. (Mitchell \& Kampfe, 1993).

Também nos anos de 1990, Everly et al. (1994) realizaram estudo para quantificar os estressores e estratégias de enfrentamento de estudantes de terapia ocupacional. Como instrumento, os pesquisadores utilizaram questionário dividido em quatro sessóes: demográfica, fonte de estresse, coping, cursos utilizados e efeitos do estresse no estudante. Participaram deste estudo 1.095 estudantes de terceiro e quarto anos do curso de terapia ocupacional, dentre os quais 972 mulheres e 113 homens, de 14 universidades dos EUA. É importante destacar que, nos EUA, diferente da modalidade de formação atual, na qual, para se tornar terapeuta ocupacional, é necessário o mestrado profissional em terapia ocupacional (American Occupational Therapy Association, 2019), nos anos de 1990, período de publicação desse artigo, estudantes também podiam fazer sua formação por meio do bacharelado, com duração de quatro anos, conforme indicado nos estudos apresentados.

Os resultados de Everly et al. (1994) apontaram que as cinco maiores fontes de estresse do curso eram as avaliaçóes das disciplinas, a quantidade de trabalhos/atividades demandadas, pouco tempo livre, longas horas de estudo e as notas alcançadas nas avaliações. Como estratégias de enfrentamento (coping), estudantes relataram fazer uso da perseverança, conversar com amigos e familiares, analisar os problemas, agir diretamente na fonte dos problemas e estudar intensamente e constantemente. De forma geral, os alunos respondiam às altas expectativas do curso, de forma funcional e produtiva.

No Brasil, em estudo mais atual, Sanches et al. (2018) buscaram avaliar a prevalência de indicadores de estresse em estudantes concluintes do curso de terapia ocupacional. 
Participaram 31 estudantes, todas mulheres e solteiras e que concluíam o curso e estavam em estágio supervisionado. Por meio do Inventário de Sintomas de Estresse para Adultos de Lipp (ISSL) e de um roteiro de entrevista semiestruturada para caracterização sóciodemográfica, verificaram que, das 31 participantes, 26 se inseriam em alguma fase de estresse. Ainda em relaçáo aos resultados, nove alunas estavam na fase de exaustão. Segundo Sanches et al. (2018), os resultados sugerem que as atividades concentradas no último ano, com destaque para o estágio curricular, o desenvolvimento de monografia final, o confronto de sua realidade, geralmente distinta, com a realidade do paciente, e as incertezas quanto ao futuro profissional, constituem um conjunto de fatores que favorece o estresse do estudante. Não obstante, os autores não discutiram em seus resultados sobre a possibilidade de outras variáveis implicarem os resultados, dentre elas, os índices de estresse mais alto geralmente verificado em mulheres brasileiras de diferentes idades (Matsukura et al., 2007).

Gilbert \& Strong (1997) realizaram pesquisa com 62 estudantes de terapia ocupacional que estavam no início do terceiro ano do curso, período prévio ao estágio curricular, em universidade da Austrália, onde a formação em terapia ocupacional é de quatro anos, buscando examinar estratégias de enfrentamento e sua relação com a ansiedade e a autoestima. Utilizaram como instrumentos da pesquisa a escala The Ways of Coping Checklist, a escala Rosenberg Self-esteem Scale e o questionário The Sixteen Personality Factor Questionnaire.

Os resultados deste estudo (Gilbert \& Strong, 1997) sugerem que estudantes ansiosos empregam a estratégia de enfrentamento de utilização de pensamento positivo, quando confrontados com uma situação que consideram provocadora de ansiedade. Os alunos que foram identificados sem ansiedade excessiva usam principalmente a estratégia focada na resoluçâo do problema, seguido por pensamento positivo e busca de apoio social. O estudo sugere a importância de identificar as características pessoais dos alunos, em particular aquelas que podem impedir a competência no trabalho de campo, no estágio curricular.

Govender et al. (2015) buscaram determinar quais os pontos estressantes do curso de terapia ocupacional e quais estratégias de enfrentamento foram empregadas por estudantes de terapia ocupacional na África do Sul. Os dados foram coletados, entre 101 estudantes de terapia ocupacional, das quais $29.3 \%$ cursavam o primeiro ano do curso, $29.3 \%$ cursavam o segundo ano, $24.2 \%$ o terceiro ano e $17.2 \%$ o quarto ano.

Os resultados apontaram que o maior nível de estresse foi apresentado pelas alunas do quarto ano; assim, considerando-se que na África do Sul a formação é de quatro anos, o último ano foi o mais estressante (Govender et al., 2015). Assim como Sanches et al. (2018), os autores explicam tal resultado pelas demandas impostas aos alunos para atender às expectativas aos requisitos deste período do curso, como estágio quase sempre em tempo integral, requisitos de pesquisa e outros compromissos acadêmicos. As estratégias de enfrentamento mais utilizadas pelos estudantes, assim como o estudo de Gilbert \& Strong (1997), eram estratégias focadas no problema, focadas no pensamento positivo e na busca de apoio social.

Witt et al. (2019) desenvolveram estudo com objetivo de determinar o nível de resiliência e estressores de estudantes de terapia ocupacional. Para alcançar tal objetivo, utilizam um questionário sociodemográfico, The Resilience Scale (RS), The Perceived 
Stress Scale (PPS) e The Health Behaviour Questionnaire (HB). Participaram 118 estudantes de terapia ocupacional.

Os resultados apontam que estudantes de terapia ocupacional estudam de 11 a 20 horas por semana, o que é considerado uma carga horária alta, visto que o curso é integral e a média de estudo dos outros cursos da mesma universidade é de 1 a 10 horas de estudo. Os alunos consideram seu nível de estresse alto e a maior fonte de estresse é a acadêmica, e para aqueles que cursam o último ano, além do estresse acadêmico, somase o estresse pessoal e social. A maioria dos estudantes tem níveis moderados de resiliência, bem como altos níveis de apoio familiar para moderar esses estressores acadêmicos, sendo a família a principal fonte de apoio social destes estudantes (Witt et al., 2019).

Os estudos deste núcleo temático correlacionam o curso de terapia ocupacional à saúde mental de estudantes de terapia ocupacional por meio de hipóteses sobre o estresse gerado pelo curso. Os estudos atribuem ao período de estágio ou à sobrecarga gerada pelas atividades acadêmicas do curso os fatores estressores. Nota-se que o estágio curricular, assim como os encargos decorrentes das atividades acadêmicas, é também fator de estresse, o que acontece também em outros cursos da área da saúde (Murakami et al., 2019; Nerdrum et al., 2009). Destaca-se que as especificidades do curso de terapia ocupacional, as particularidades de estudantes que optam por esta formação, assim como questóes trazidas por estas últimas, não foram variáveis consideradas ao relacionarem o curso à saúde mental deste estudante.

\section{Saúde mental de estudantes de terapia ocupacional vista em paralelo à saúde mental de estudantes do campo da saúde}

Cavestro \& Rocha (2006) desenvolveram pesquisa para identificar e comparar a prevalência de transtorno depressivo e risco de suicídio entre estudantes de medicina, fisioterapia e terapia ocupacional de uma faculdade particular da Regiáo Sudeste brasileira. Foram entrevistados 342 alunos: 90 do curso de fisioterapia, 213 do curso de medicina e 39 da terapia ocupacional. O número da amostragem foi calculado por cota, de acordo com o número de alunos inscritos em cada curso. No curso de terapia ocupacional todos os participantes eram do sexo feminino. Como instrumento, utilizaram o Mini International Neuropsychiatric Interview (MINI), que é uma entrevista por meio da qual se obtém o diagnóstico psiquiátrico e risco de suicídio.

Como resultado, a prevalência de depressão maior foi de $10,5 \%$ entre os estudantes, mas as alunas de terapia ocupacional tiveram uma prevalência maior $(28,2 \%)$ que alunos de medicina $(8,9 \%)$ e alunos de fisioterapia $(6,7)$. Em relação ao risco de suicídio, as estudantes de terapia ocupacional apresentaram risco maior $(25,6 \%)$ em relação aos estudantes de medicina $(7,5 \%)$ e estudantes de fisioterapia $(7,8 \%)$. Estimou-se que a chance de alunas de terapia ocupacional apresentarem depressão é 3,6 vezes maior que as chances dos alunos de medicina e de fisioterapia. Em relação às chances de suicídio, as alunas de terapia ocupacional apresentaram risco 3,7 vezes maior que os outros alunos da pesquisa. Os autores discutem o fato dos objetivos do curso exercerem influência na escolha da profissão por parte do aluno, o que poderia levar a pensar em um processo de seleção de natureza desconhecida, que justificasse maior número de pessoas com transtornos depressivos entre tais estudantes. Outra explicação para os resultados 
observados é que as alunas de terapia ocupacional teriam maior facilidade em comentar aspectos de seu funcionamento psicológico, quando comparadas a estudantes de outros cursos (Cavestro \& Rocha, 2006).

Outro estudo que aponta as estudantes de terapia ocupacional com prevalência maior de transtorno mental, em relação a outros cursos de graduação, é a pesquisa de dissertaçáo de mestrado de Arantes (2018). A autora analisou a influência do Transtorno Mental Comum (TMC) sobre a Qualidade de Vida (QV) de graduandos de ciências da saúde de uma instituição pública de ensino brasileira. Este estudo apontou as estudantes terapeutas ocupacionais com maior prevalência de TMC, em comparação a estudantes de outros seis cursos, participantes da amostragem.

A pesquisa de Arantes (2018) foi desenvolvida com participação de 195 estudantes do curso de Biomedicina, 235 do curso de Educação Física, 308 de Enfermagem, 311 de Fisioterapia, 511 de Medicina, 251 de Nutrição e 185 graduandos do curso de terapia ocupacional. O número da amostra por curso respeitou a distribuição também proporcional de discentes por curso de ciências da saúde. Foi utilizado um questionário sociodemográfico e acadêmico desenvolvido pela autora, além do Self Reporting Questionnaire e o World Health Organization Quality of Life-bref (WHOQOL-bref).

Como resultado, o curso de terapia ocupacional apresentou a maior prevalência de TMC com 56\% entre os alunos. Os principais sintomas relatados por estudantes foram sensação de nervosismo, tensão e preocupação, seguidos do sintoma somático dormir mal e da dificuldade em tomar decisóes. Segundo a autora, os resultados apontam como a ocorrência de TMC pode afetar a QV dos estudantes, assim como a análise dos sintomas descritos podem auxiliar intervençôes que permitam a melhoria do bem-estar do estudante e sua permanência na universidade (Arantes, 2018).

Murakami et al. (2019), em estudo sobre estresse psicológico entre estudantes da área da saúde, também encontraram índice maior entre estudantes de terapia ocupacional em relaçáo a estudantes de outros cursos. Participaram deste estudo 9 estudantes de terapia ocupacional, 18 de fisioterapia, 15 de fonoaudiologia, 45 de medicina e 15 de nutriçáo de uma mesma instituição de ensino, pública, situada na Região Sudeste brasileira. O número de alunos por curso foi definido estatisticamente de forma estratificada, com compartilhamento proporcional do número total de alunos de cada curso e em cada período. Os participantes responderam a um questionário com dados sociais e demográficos e ao Inventário de Sintomas de Stress para Adultos de Lipp (ISSL).

A maior incidência de estresse foi encontrada no curso de terapia ocupacional, com $88 \%$ dos estudantes, enquanto outros cursos apresentaram uma incidência aproximada de $60 \%$. Não houve diferenças estatisticamente significantes entre os cursos, sendo destacado que, na área da saúde, a presença de estresse é independente da natureza do curso (Murakami et al., 2019).

Nerdrum et al. (2009) apontam resultados diferentes dos anteriores em relação à estudante de terapia ocupacional. Foi realizado estudo entre 126 estudantes, todas do gênero feminino, dos cursos de enfermagem (57,5\% das alunas matriculadas), 76 de fisioterapia (85\%) e 33 de terapia ocupacional (85,5\%), de universidade norueguesa, buscando investigar o estresse psicológico entre elas. Para tal pesquisa, utilizaram The StudData Questionnaires e General Health Questionnaire 12 como instrumentos, aplicados no início e no fim da graduação. 
Ao contrário dos resultados das pesquisas anteriores, a estudante de terapia ocupacional teve o nível de estresse psicológico menor em relação aos outros cursos. A aluna de enfermagem foi a que apresentou maior nível de estresse psicológico desde o início até a conclusão do curso. As estudantes de terapia ocupacional relataram o nível mais baixo e as estudantes de enfermagem relataram o mais alto grau de sobrecarga. Nerdrum et al. (2009) apontam ainda que a clareza em relação à estrutura do programa de ensino foi uma variável significativa na explicação da angústia das alunas, ou seja, a estrutura e o programa do curso, quando não compreendidos pelas estudantes, podem ser geradores de angústia entre as alunas (Nerdrum et al., 2009).

Souza et al. (2011) buscaram detectar prevalência indicativa de anorexia e bulimia nervosas em universitárias dos cursos de nutrição, enfermagem, psicologia e terapia ocupacional, bem como avaliar a autoimagem corporal, tomando-se como amostra 352 estudantes do sexo feminino de uma instituição de ensino privado do Centro-Oeste brasileiro, sendo 51 (14,49\% das alunas matriculadas no curso) de enfermagem, 153 (43,47\%) de nutrição, 133 (37,78\%) de psicologia e 15 (4,26\%) de terapia ocupacional. Como instrumentos, utilizaram questionário sociodemográfico, o Eating Attitudes Test (EAT-26) - Teste de Atitudes Alimentares - e o Body Shape Questionnaire (BSQ) Questionário de Imagem Corporal.

Como resultado, o curso de nutrição apresentou maior prevalência de indicativos de sintomatologia relacionada aos TA, em relação aos cursos de terapia ocupacional, enfermagem e psicologia. Os autores ressaltam a importância de desenvolvimento de programas preventivos para os TA nas universidades, já que as alunas com depressão, obesas ou com preocupação grave com a imagem corporal constituem grupos de risco para tais transtornos.

Diante dos resultados apresentados, é possível constatar que todos os estudos nacionais, com exceçâo de um deles, indicaram maiores índices de sofrimento psíquico em estudantes de terapia ocupacional, comparados com alunos de outros cursos da área da saúde. $\mathrm{O}$ único estudo internacional apresentado traz resultado diverso. Apesar do número de participantes do curso de terapia ocupacional, em todas as pesquisas apresentadas, ser menor em relação aos outros cursos, isto é justicado, com exceção do estudo de Souza et al. (2011), que não traz esta justificativa, como número proporcional ao número de alunos matriculados no curso. As discussóes dos resultados são feitas por meio de hipóteses e, especificamente em relação a estudantes de terapia ocupacional, as considerações são ainda superficiais, o que indica a necessidade de novos estudos que possam aprofundar nesta identificação de aspectos do curso e deste grupo de estudantes, que levem à compreensão do sofrimento psíquico da aluna de terapia ocupacional.

\section{Variáveis associadas à saúde mental de estudantes de terapia ocupacional}

Bonsaksen (2015) pesquisou a saúde mental associada a fatores relacionados a dados sociodemográficos, principalmente em relação ao gênero, associados à autoeficácia geral e autoestima em 148 estudantes de terapia ocupacional da Noruega. O grupo de estudantes participantes foi dividido em três: primeiro ano (10 homens e 43 mulheres), segundo ano (12 homens e 37 mulheres) e terceiro ano (9 homens e 37 mulheres). É importante destacar que na Noruega o curso de terapia ocupacional tem duração de três 
anos. Como instrumentos, foram utilizados questionário sociodemográfico, General Self-Efficacy Scale (GSE) e Rosenberg Self-Esteem Scale (RSES).

Os resultados indicaram que a maior autoeficácia é associada ao sexo masculino, assim como à satisfaçáo com o curso, ao fato de ter mais horas de estudo e à autoestima. $\mathrm{O}$ autor ressalta que tais fatores parecem ser indicadores de uma melhor saúde mental e, como são associados ao sexo masculino, alertam para a possibilidade de que as alunas possam ter risco maior em relação ao sofrimento psíquico. Dessa forma, dado haver relaçôes estreitas entre esses resultados e a saúde mental, é necessária atenção geral às autopercepções, a aspectos relacionados às horas dispensadas ao estudo pelos alunos (homens e mulheres), assim como à importância da satisfação em relação ao curso (Bonsaksen, 2015).

Kotera et al. (2019) realizaram pesquisa em universidade no Reino Unido e buscaram a elucidação das relaçóes entre a saúde mental, atitudes frente à saúde mental, à autocrítica, à autocompaixão e à identidade de cuidador. Participaram 165 estudantes de período integral dos cursos de terapia ocupacional e aconselhamento ${ }^{2}$, dos quais $90 \%$ eram mulheres, com idade entre 17 a 52 anos. Os pesquisadores utilizaram como instrumentos as seguintes escalas: Depression, Anxiety, and Stress Scale 21 (DASS 21), Attitudes Towards Mental Health Problems (ATMHP), Forms ofSelfCriticising/Attacking\& Self-Reassuring Scale (FSCSR), Self-Compassion Scale-Short Form e Role Identity Scale (RIS).

Os resultados revelaram que a saúde mental estava associada a atitudes como autocrítica, autoconfiança e autocompaixão. A autocrítica e o sentimento de vergonha foram preditores significativos de problemas de saúde mental nesses estudantes. Contrariando a hipótese dos autores, a saúde mental não estava relacionada à identidade de cuidador (Kotera et al., 2019).

McCombie (2019) realizou estudo com o objetivo de verificar o uso abusivo de álcool e comportamento de risco decorrente do consumo de álcool entre estudantes de terapia ocupacional. A amostra foi constituída por 285 estudantes de terapia ocupacional associados da American Occupational Therapy Association (AOTA). Foi utilizado como instrumento o Alcohol Use Disorders Identification Test (AUDIT), para identificar níveis de álcool prejudicial e de consumo perigoso. Além disso, utilizou um checklist de itens de comportamento arriscado, formulado com base em revisão da literatura sobre estudantes universitários e consumo de álcool.

Quase todos os estudantes relataram ter o hábito de consumir bebida alcoólica, porém, a quantidade e frequência variavam entre eles. Os resultados apontam que dois a cada cinco estudantes apresentam tendência ao uso abusivo do álcool. Com base nesses resultados, a pontuação do AUDIT aconselha a educação básica sobre o álcool para a maioria (85\%) desses estudantes (McCombie, 2019).

Pinho (2018) realiza pesquisa sobre o perfil do uso de álcool e tabaco e seus fatores associados, entre os estudantes do curso de terapia ocupacional de uma universidade pública do Sudeste brasileiro. Participaram do estudo 111 estudantes, sendo a maioria do sexo feminino $(83,8 \%)$, com idade entre 18 e 24 anos, pertencentes à classe econômica B1 /B2 e, em relação à religião, eram evangélicos/protestantes ou católicos.

${ }^{2} \mathrm{O}$ Aconselhamento é uma profissão, inexistente no Brasil, dedicada a ajudar pessoas com problemas situacionais e de longo prazo. 
Os participantes responderam a um questionário proposto pela Secretaria Nacional de Políticas sobre Drogas (SENAD) para o I Levantamento Nacional sobre o Uso de Álcool e Outras Drogas entre Universitários das 27 capitais brasileiras, realizado em 2009.

Os resultados encontrados mostraram que beber em binge, ou seja, beber muito em um curto período de tempo, nos últimos 12 meses ou nos últimos 30 dias, é mais frequente que o estudo de referência nacional. Foi feita associação entre a religião a variável desfecho binge drinking no ano, sugerindo que entre os universitários pesquisados que não possuíam uma religiáo há uma maior chance de encontrar aqueles que beberam muito, de forma episódica, nos últimos 12 meses (Pinho, 2018).

O núcleo temático apresenta, por um lado, uma variedade de indicadores, como: autoeficácia, autocompaixão, autoestima e fatores associados, como satisfação com o curso, ter mais horas de estudo, que são identificados como fatores que contribuem positivamente à saúde mental deste grupo de estudantes. Por outro lado, a autocrítica e a vergonha, e o uso abusivo de álcool são identificados como preditivos de problemas de saúde mental. Apesar de se tratar de informaçóes úteis sobre a saúde mental de estudantes de terapia ocupacional, faz-se necessária a realização de estudos que indiquem a direção causal de tais fatores, assim como busquem elucidar, de forma mais sistemática, os fatores protetivos e fatores de risco à saúde mental de estudante de terapia ocupacional.

\section{Discussáo}

Esta revisão compreende o período de 26 anos, com publicação inicial em 1993 até 2019. O aumento de publicação sobre a saúde mental de estudantes de terapia ocupacional, nos últimos anos, vai ao encontro do aumento das taxas de transtorno mental entre os estudantes universitários, destacando a saúde mental como uma crescente preocupação de saúde pública nas universidades (Fórum Nacional de PróReitores de Assuntos Estudantis, 2019; Liu et al., 2019; McLafferty et al., 2017; Tran et al., 2017). Além da prevalência e efeitos deletérios de problemas de sofrimento psíquico entre os estudantes universitários, há um alerta entre os pesquisadores e a sociedade em geral, quanto ao aumento da taxa de suicídio entre esta populaçáo (Fórum Nacional de Pró-Reitores de Assuntos Estudantis, 2019; Lima et al., 2019), o que justificaria o aumento de produção de pesquisas sobre a saúde mental do universitário.

No Brasil, na conjuntura verificada nos últimos anos, as universidades públicas brasileiras enfrentam processo de precarização com redução de verbas para sua manutenção básica, além de não receberem subsídios necessários para o atendimento a estudantes que se encontram em desvantagem econômica, educacional e/ou discriminados e vitimados pela exclusão social. Assim, por exemplo, faltam recursos para estudantes com essas desvantagens, sendo necessário que muitos deles/delas trabalhem, tendo jornada dupla de estudo-trabalho, podendo trazer uma sobrecarga que afeta a saúde mental destes/destas jovens (Tombolato, 2005).

As pesquisas apresentadas neste estudo são investigaçóes quantitativas, o que reforça a indicação de que campos de significados em relação à saúde mental de estudante de terapia ocupacional continuam em aberto para serem explorados, sendo um campo fértil para pesquisas de natureza qualitativa. Outro dado importante a ser destacado é a variedade de questionários e escalas utilizada. Para além dos questionários de cunho 
sociodemográfico, os 15 estudos apresentaram uma variedade de 22 escalas/questionários, havendo a repetição de apenas dois desses instrumentos. Ainda que este dado confirme uma vasta gama de medidas e instrumentos confiáveis e apropriados para o estudo da saúde mental da estudante de terapia ocupacional, traz questão referente à variedade de instrumentos na pesquisa de um mesmo tema, o que, dentre outros aspectos, dificulta o diálogo mais pertinente entre os resultados.

Nesses 26 anos, foram várias as mudanças nos cursos de formação em terapia ocupacional, por exemplo: obrigatoriedade do mestrado profissional como formação básica para o terapeuta ocupacional nos EUA (American Occupational Therapy Association, 2019), obrigatoriedade de aumento do tempo de integralização de cursos com mais de 3.600 horas para cinco anos, no Brasil (Brasil, 2009), além de mudanças das matrizes curriculares dos cursos (Barba et al., 2012). No entanto, verifica-se que, desde as primeiras publicações (Gilbert \& Strong, 1997; Mitchell \& Kampfe, 1993; Everly et al., 1994) até as publicaçóes atuais, ainda que com as mudanças ocorridas, a transição de aluno de um ambiente acadêmico para um ambiente clínico ainda pode ser estressante e gerar prejuízos à saúde mental de estudantes, como continua apontando a literatura (Govender et al., 2015; Sanches et al., 2018).

O padrão mínimo para educação em terapia ocupacional, segundo a World Federation of Occupational Therapy (World Federation of Occupational Therapists, 2016), é de 1000 horas de colocaçóes práticas para garantir a integração da teoria à prática e se refere ao tempo que cada aluno passa com pessoas como usuários/pacientes/clientes que podem ser indivíduo, família, grupo ou comunidade, nos campos de prática, que podem ser variados (instituiçóes públicas, clínicas, escolas etc.), desenvolvendo um processo em terapia ocupacional. No contexto nacional, as Diretrizes Curriculares Nacionais (DCN) de terapia ocupacional instituem que as atividades práticas específicas da terapia ocupacional devem ser desenvolvidas gradualmente desde o início do curso. Porém, isso parece não ser suficiente para evitar o estresse da aluna e do aluno na passagem para o estágio curricular, período que pode ocasionar transtornos emocionais nestas/nestes estudantes (Sanches et al., 2018). É importante destacar que o estresse decorrente do estágio profissionalizante não é exclusivo da aluna e do aluno de terapia ocupacional. Estudos de outros cursos da área da saúde apontam este momento de estágio como estressante também para outros estudantes (Pereira et al., 2014; Querido et al., 2016).

Os estudos sugerem que seja feito suporte emocional às alunas e aos alunos pela instituição de ensino, supervisores e professores (Sanches et al., 2018), que seja conhecido o coping utilizado por elas e por eles, identificando o que pode ser adaptado pelos programas de apoio às/aos estudantes (Govender et al., 2015), identificando também as características pessoais das alunas e dos alunos, que possam impedir a competência no trabalho de campo e, a partir daí, desenvolver programa para auxílio nas habilidades deficitárias (Gilbert \& Strong, 1997).

Vale destacar que, durante o estágio, a/o estudante que está terminando seu curso experimenta também a transição entre educação e trabalho. Estudos apontam que o fator estressor mais frequente entre as formandas e os formandos é o medo do futuro profissional (Amr et al., 2011; Vicente, 2014). Além disso, esta passagem de estudante para profissional envolve a perda da condição de aluno/aluna e do apoio da universidade, além da frequente perda da influência da família, pela necessidade do/da jovem construir 
sua própria identidade, o que pode gerar insegurança, apatia, desorganização e consequente sofrimento psíquico (Geirdal et al., 2019). Compreende-se que colocar foco neste período de conclusão do curso pode revelar dimensóes importantes que podem ser abordadas com mais elementos ao longo de toda a formação. Nesse sentido, estudos futuros podem contribuir nesta ampliação da compreensão.

Altos níveis de estresse nessa população está relacionado ao uso abusivo de álcool e outras drogas e à prevalência de transtornos mentais (Dennhardt \& Murphy, 2011; Kenney et al., 2018). A prevalência de transtornos mentais e uso abusivo de álcool e outras substâncias psicoativas entre as alunas e os alunos de terapia ocupacional, assim como fatores de risco e proteção foram pouco explorados, apesar do impacto negativo que tais transtornos causam na vida e nas atividades acadêmicas da/do estudante.

Sobre as variáveis sociodemográficas, é importante destacar que são significativamente associadas à prevalência de transtornos mentais (Gorn et al., 2005; Klose \& Jacobi, 2004). Estudos apontam que nível socioeconômico e gênero estáo associados a uma probabilidade significativamente aumentada de transtornos mentais (Andrade et al., 2012; Schwartz et al., 2011). Em relação ao gênero, o estudo de Bonsaksen (2015) apresentou que fatores como maior eficácia e satisfação com o curso de terapia ocupacional estão associados ao sexo masculino. Tais fatores, segundo o autor, seriam indicadores de melhor saúde mental, ou seja, o estudante do sexo masculino apresenta risco menor de sofrimento psíquico.

Esta discussão faz sentido ao nos reportarmos ao fato da grande maioria de estudantes de terapia ocupacional ser mulheres, conforme mostram os estudos selecionados, indicando a importância de maior contextualização e aprofundamento sobre a questão do feminino e, para além (e a par com a realidade), com as questóes de gênero e seus desdobramentos nos percursos de vida e de saúde mental de estudantes de terapia ocupacional. Complementarmente, indica-se a necessidade de colocar foco na diversidade de estruturas, de acesso e de vivências, especialmente aquelas impostas aos diferentes grupos vulnerabilizados e/ou que não são representados por um padrão normativo comumente considerado nas análises de resultados de investigaçóes aqui abordadas.

Em termos nacionais, sabe-se que, atualmente, a universidade apresenta uma nova configuração, tornando-se mais representativa da sociedade em que está inserida e, consequentemente, com diversos problemas por ela enfrentados. A expansão da educação superior brasileira pelo Programa de Apoio a Planos de Reestruturação e Expansão das Universidades Federais (REUNI), a partir de 2007, teve como objetivo ampliar o acesso de uma maior proporção da juventude brasileira à educação superior, especialmente pelo baixo número de estudantes nesse nível de ensino. Além disso, a democratização e expansão do acesso, estratégias de garantia de permanência de grupos historicamente discriminados nas instituiçóes universitárias, como negros, índios e pessoas em condiçóes socioeconômicas precárias, foram colocadas em pauta por meio da implementação de políticas de açôes afirmativas nas universidades. Dessa forma, reconhecer as especificidades das condiçóes da aluna e do aluno de terapia ocupacional e considerá-los no processo de investigação é qualificar a pesquisa da saúde mental desta/deste estudante.

Alguns estudos apontam que o grupo de estudantes de terapia ocupacional é mais afetado em prejuízos à sua saúde mental se comparado a alunos e alunas de outros cursos 
(Arantes, 2018; Cavestro \& Rocha, 2006; Murakami et al., 2019). É interessante notar que os estudos que trazem a Terapia Ocupacional como curso com maior número de estudantes com transtorno mental comum (Arantes, 2018), com depressão (Cavestro \& Rocha, 2006) ou, ainda, com estresse psicológico (Murakami et al., 2019) são frutos de pesquisas nacionais, realizadas em contexto brasileiro.

Este resultado nos remete mais uma vez à questão relacionada ao contexto social e a trajetórias diversas deste/desta estudante, à caracterização do perfil deste grupo de jovens ou, ainda, aos modos de subjetivaçáo de estudantes brasileiros de terapia ocupacional. Estas variáveis se associam à estrutura do curso - já que, de acordo com estudos apresentados, o curso de terapia ocupacional traz sobrecarga -, podendo trazer prejuízos à saúde mental de estudantes. Dessa forma, compreende-se que trazer luz às dimensóes do contexto de vida permite, dentre outras, a possibilidade de abordar a saúde mental de forma mais ampla e em diálogo com a perspectiva psicossocial.

\section{Consideraçóes Finais}

A revisão de escopo sobre a saúde mental de estudante de terapia ocupacional aponta a carga horária de colocaçóes práticas de 1000 horas no decorrer do curso e a imersão da aluna e do aluno no campo de estágio como fonte de estresse e sofrimento psíquico entre estudantes. Outras dimensóes foram apresentadas e discutidas com base em categorias identificadas, como os índices de sofrimento psíquico das alunas e dos alunos de terapia ocupacional serem maiores que os de alunos de outros cursos da área da saúde, assim como indicadores e fatores associados à saúde mental de estudante de terapia ocupacional.

As pesquisas apresentadas nesta revisão são frutos de investigação quantitativa que não abrangem significados atribuídos por alunas e alunos em relação à saúde mental, mostrando a relevância de investigaçóes qualitativas e mistas nos estudos sobre o tema. Pesquisas nesta direçáo podem trazer elementos novos para a compreensáo de um fato importante e preocupante, para os envolvidos com a formação de terapeutas ocupacionais, que é a prevalência de sofrimento psíquico entre estudantes de terapia ocupacional.

Além disso, destaca-se a necessidade de considerar algumas variáveis importantes que fazem parte da caracterização deste grupo de estudantes, quais sejam: a importância de maior contextualização e aprofundamento sobre as questóes que envolvem as mulheres, sobre as questóes de gênero, de raça/etnia, condição socioeconômica, diversidade de estruturas e acessos à universidade, além de seus reflexos nos percursos de vida e na saúde mental destas/destes estudantes.

Como discutido a partir dos resultados obtidos neste estudo, aponta-se para a premente necessidade de que estudos futuros sobre o tema se debrucem sobre as especificidades do curso de terapia ocupacional, das particularidades do grupo de estudantes, elucidando os diferentes fatores e indicadores associados à saúde mental deste grupo.

Destaca-se, ainda, a relevância de tais estudos para os interessados em estudantes, na formação da/do terapeuta ocupacional, no futuro profissional e na qualidade de atenção e cuidado para a população-alvo desta profissão, assim como para a promoção de açóes que forneçam apoio para as/os estudantes em sofrimento psíquico. 


\section{Referências}

American Occupational Therapy Association - AOTA. (2019). Results from RA 2019 springs meetings: motions include OT and OTA entry level mandates. Recuperado em 20 de maio de 2020, de https://www.aota.org/Publications-News/AOTANews/2019/Results-RA-Spring-Meeting-MotionsInclude-Entry-Level-Mandates.aspx

Amr, A., El-Gilany, A. H., El-Moafee, H., Salama, L., \& Jimenez, C. (2011). Stress among Mansoura (Egypt) baccalaureate nursing students. The Pan African Medical Journal, 8(1), 1-9. http://dx.doi.org/10.4314/pamj.v8i1.71083.

Andrade, L. H., Wang, Y. P., Andreoni, S., Silveira, C. M., Alexandrino-Silva, C., Siu, E. R., Nishimura, R., Anthony, J. C., Gattaz, W. F., Kessler, R. C., \& Viana, M. C. (2012). Mental disorders in megacities: findings from the São Paulo megacity mental health survey, Brazil. PLoS One, 7(2), e31879.

Anversa, A. C., Santos Filha, V. A. V. D., Silva, E. B. D., \& Fedosse, E. (2018). Qualidade de vida e o cotidiano acadêmico: uma reflexão necessária. Cadernos Brasileiros de Terapia Ocupacional, 26(3), 626-631.

Arantes, A. P. (2018). Qualidade de vida e transtorno mental comum em graduandos de ciências da saúde (Dissertação de mestrado). Universidade do Triângulo Mineiro, Uberaba.

Barba, P. C. D. S. D., Silva, R. F. D., Joaquim, R. H. V. T., \& Brito, C. M. D. D. (2012). Formação inovadora em terapia ocupacional. Interface: Comunicação, Saúde, Educação, 16(42), 829-842. http://dx.doi.org/10.1590/S1414-32832012000300019.

Bardin, L. (2011). Análise de conteúdo. São Paulo: Edições 70.

Bonsaksen, T. (2015). Predictors of general self-efficacy and self-esteem in occupational therapy students: A cross-sectional study. Occupational Therapy in Mental Health, 31(3), 298-310. http://dx.doi.org/10.1080/0164212X.2015.1055536.

Brasil. (2009, 11 de março). Parecer CNE/CES n. 213/2008. Dispóe sobre carga horária mínima e procedimentos relativos à integralização e duração dos cursos de graduação em Biomedicina, Ciências Biológicas, Educação Física, Enfermagem, Farmácia, Fisioterapia, Fonoaudiologia, Nutrição e Terapia Ocupacional, bacharelados, na modalidade presencial. Diário Oficial [da] República Federativa do Brasil, Brasília. Recuperado em 20 de maio de 2020, de http://portal.mec.gov.br/cne/arquivos/pdf/2008/pces213_08.pdf

Carleto, C. T., Moura, R. C. D., Santos, V. S., \& Pedrosa, L. A. K. (2018). Adaptação à universidade e transtornos mentais comuns em graduandos de enfermagem. Revista Eletrônica de Enfermagem, 20, 1-11. http://dx.doi.org/10.5216/ree.v20.43888.

Cavestro, J. M., \& Rocha, F. L. (2006). Prevalência de depressão entre estudantes universitários. Jornal Brasileiro de Psiquiatria, 55(4), 264-267. http://dx.doi.org/10.1590/S0047-20852006000400001.

Claudino, A. C. S. O., Santos Braz, F., de Souza, L. P., Rubio, L. S., da Silva, N. C., \& Pereira, A. V. (2019). Relato de experiência: ansiedade, estresse e Depressão no âmbito universitário. Revista Interdisciplinar Pensamento Científico, 5(5), 1670-1686.

Dennhardt, A. A., \& Murphy, J. G. (2011). Associations between depression, distress tolerance, delay discounting, and alcohol-related problems in European American and African American college students. Psychology of Addictive Behaviors, 25(4), 595-604.

Everly, J. S., Poff, D. W., Lamport, N., Hamant, C., \& Alvey, G. (1994). Perceived stressors and coping strategies of occupational therapy students. The American Journal of Occupational Therapy, 48(11), 1022-1028.

Fond, G., Bourbon, A., Lançon, C., Boucekine, M., Micoulaud-Franchi, J. A., Auquier, P., \& Boyer, L. (2019). Psychiatric and psychological follow-up of undergraduate and postgraduate medical students: prevalence and associated factors. Results from the national BOURBON study. Psychiatry Research, 272, 425-430. http://dx.doi.org/10.1016/j.psychres.2018.12.174. 
Fórum Nacional de Pró-Reitores de Assuntos Estudantis - FONAPRACE. Associação Nacional dos Dirigentes das Instituiçóes de Ensino Superior - ANDIFES. (2016). IV Pesquisa do Perfil Sócioeconômico e Cultural dos Estudantes de Graduação das s InstituiçôesFederais de Ensino Superior Brasileiras 2014. Uberlândia: Universidade Federal de Uberlândia. Recuperado em 2 de setembro de 2020, de http://www.andifes.org.br/wp-content/uploads/2017/11/Pesquisa-de-Perfil-dosGraduansodas-IFES_2014.pdf

Fórum Nacional de Pró-Reitores de Assuntos Estudantis - FONAPRACE. Associação Nacional dos Dirigentes das Instituiçôes de Ensino Superior - ANDIFES. (2019). V Pesquisa Nacional de Perfil Socioeconômico e Cultural dos(as) Graduandos (as) das IFES - 2018. Uberlândia: Universidade Federal de Uberlândia. Recuperado em 2 de setembro de 2020, de http://www.andifes.org.br/wpcontent/uploads/2019/05/V-Pesquisa-do-PerfilSocioecon\%C3\%B4mico-dos-Estudantes-deGradua\%C3\%A7\%C3\%A3o-das-UniversidadesFederais-1.pdf

Geirdal, A. O., Nerdrum, P., \& Bonsaksen, T. (2019). The transition from university to work:what happens to mental health? A longitudinal study. BMC Psychology, 7(1), 1-10. http://dx.doi.org/10.1186/s40359-019-0340-x.

Gilbert, J., \& Strong, J. (1997). Coping strategies employed by occupationa therapy students anticipating fieldwork placement. Australian Occupational Therapy Journal, 44(1), 30-40. http://dx.doi.org/10.1111/j.1440-1630.1997.tb00751.x.

Gonçalves, R., \& Ambar, G. (2015). A questão racial, a universidade e a (in) consciência negra. Lutas Sociais, 19(34), 202-213. http://dx.doi.org/10.23925/ls.v19i34.25767.

Gorn, S. B., Tiburcio Sainz, M., \& Medina-Mora Icaza, M. (2005). Variables demográficas asociadas con la depresión: diferencias entre hombres y mujeres que habitan en zonas urbanas de bajos ingresos. Salud Mental, 28(6), 33-40.

Govender, P., Mkhabela, S., Hlongwane, M., Jali, K., \& Jetha, C. (2015). OT student's experiences of stress and coping. South African Journal of Occupational Therapy, 45(3), 34-39.

Grace, M. K. (2018). Depressive symptoms, burnout, and declining medical career interest among undergraduate pre-medical students. International Journal of Medical Education, 9, 302-308. http://dx.doi.org/10.5116/ijme.5be5.8131.

Ibrahim, A. K., Kelly, S. J., Adams, C. E., \& Glazebrook, C. (2013). A systematic review of studies of depression prevalence in university students. Journal of Psychiatric Research, 47(3), 391-400.

Kenney, S. R., DiGuiseppi, G. T., Meisel, M. K., Balestrieri, S. G., \& Barnett, N. P. (2018). Poor mental health, peer drinking norms, and alcohol risk in a social network of first-year college students. Addictive Behaviors, 84, 151-159.

Klose, M., \& Jacobi, F. (2004). Can gender differences in the prevalence of mental disorders be explained by sociodemographic factors? Archives of Women's Mental Health, 7(2), 133-148.

Kotera, Y., Green, P., \& Sheffield, D. (2019). Mental health of therapeutic students: relationships with attitudes, self-criticism, self-compassion, and caregiver identity. British Journal of Guidance \& Counselling. In press. http://dx.doi.org/10.1080/03069885.2019.1704683.

Leão, A. M., Gomes, I. P., Ferreira, M. J. M., \& Cavalcanti, L. P. D. G. (2018). Prevalência e fatores associados à depressão e ansiedade entre estudantes universitários da área da saúde de um grande centro urbano do Nordeste do Brasil. Revista Brasileira de Educação Médica, 42(4), 55-65.

Lima, S. O., Lima, A. M. S., Barros, E. S., Varjão, R. L., Santos, V. F., Varjão, L. L., Mendonça, A. K. R. H., Nogueira, M. S., Deda, A. V., Jesus, L. K. A., \& Santana, V. R. (2019). Prevalência da depressão nos acadêmicos da área de saúde. Psicologia, 39, e187530. http://dx.doi.org/10.1590/19823703003187530 .

Liu, C. H., Stevens, C., Wong, S. H., Yasui, M., \& Chen, J. A. (2019). The prevalence and predictors of mental health diagnoses and suicide among US college students: implications for addressing disparities in service use. Depression and Anxiety, 36(1), 8-17. 
Matsukura, T. S., Marturano, E. M., Oishi, J., \& Borasche, G. (2007). Estresse e suporte social em mães de crianças com necessidades especiais. Revista Brasileira de Educação Especial, 13(3), 415-428.

McCombie, R. P. (2019). Age of first drink, first alcohol intoxication, and alcohol abuse behaviors among occupational therapy students. The Open Journal of Occupational Therapy, 7(2), 1-14. http://dx.doi.org/10.15453/2168-6408.1555.

McLafferty, M., Lapsley, C. R., Ennis, E., Armour, C., Murphy, S., Bunting, B. P., Bjourson, A. J., Murray, E. K., \& O’Neill, S. M. (2017). Mental health, behavioural problems and treatment seeking among students commencing university in Northern Ireland. PLoS One, 12(12), e0188785.

Mitchell, M. M., \& Kampfe, C. M. (1993). Student coping strategies and perceptions of fieldwork. The American Journal of Occupational Therapy, 47(6), 535-540.

Moher, D., Liberati, A., Tetzlaff, J., \& Altman, D. G. (2009). Preferred reporting items for systematic. reviews and meta-analyses: the PRISMA statement. PLoS Medicine, 6(6), e1000097. http://dx.doi.org/10.1371/journal.pmed.1000097.

Murakami, K., Panúncio-Pinto, M. P., Santos, J. L. F., \& Troncon, L. E. D. A. (2019). Psychological stress in students from undergraduate courses in health professions: contribution to promote mental health/Estresse psicologico em estudantes de cursos de graduacao da area da saude: subsidios para promocao de saude mental. Revista de Medicina, 98(2), 108-114.

Nerdrum, P., Rust Øen, T., \& Helge RØnnestad, M. (2009). Psychological distress among nursing, physiotherapy and occupational therapy students: a longitudinal and predictive study. Scandinavian Journal of Educational Research, 53(4), 363-378.

Orellana, J. D. Y., Ribeiro, M. R. C., Barbieri, M. A., Saraiva, M. C., Cardoso, V. C., Bettiol, H., Silva, A. A. M., Barros, F. C., Gonçalves, H., Wehrmeister, F. C., Menezes, A. M. B., Del-Ben, C. M., \& Horta, B. L. (2020). Mental disorders in adolescents, youth, and adults in the RPS Birth Cohort Consortium (Ribeirão Preto, Pelotas and São Luís), Brazil. Cadernos de Saúde Pública, 36(2), e00154319. http://dx.doi.org/10.1590/0102-311x00154319.

Pereira, F. G. F., Nunes, L. N., Miranda, M. C., \& Caetano, J. A. (2014). Assessment of stress in the inclusion of nursing students in hospital practice. Investigacion y Educacion en Enfermeria, 32(3), 430-437.

Peters, M. D. J., Godfrey, C. M., \& Khalil, H. (2015). Guidance for conducting systematic scoping reviews. International Journal of Evidence-Based Healthcare, 13(3), 141-146.

Pinho, M. C. (2018). Uso de álcool e tabaco entre universitários de terapia ocupacional de uma universidade pública (Dissertação de mestrado). Universidade Federal do Espírito Santo, Vitória.

Querido, I. A., Naghettini, A. V., Orsini, M. R. D. C. A., Bartholomeu, D., \& Montiel, J. M. (2016). Fatores associados ao estresse no internato médico. Revista Brasileira de Educação Médica, 40(4), 565573.

Sanches, B. P., Silva, N. R. D., \& Silva, M. L. (2018). Avaliação do estresse em estudantes concluintes de terapia ocupacional. Cadernos Brasileiros de Terapia Ocupacional, 26(1), 153-161. http://dx.doi.org/10.4322/2526-8910.ctoAO1025.

Schwartz, R., Lent, J., \& Geihsler, J. (2011). Gender and diagnosis of mental disorders: implications for mental health counseling. Journal of Mental Health Counseling, 33(4), 347-358.

Souza, A. A., Souza, J. C., Hirai, E. S., Luciano, H. A., \& Souza, N. (2011). Estudo sobre a anorexia e bulimia nervosa em universitárias. Psicologia: Teoria e Pesquisa, 27(2), 195-198. http://dx.doi.org/10.1590/S0102-37722011000200012.

Tombolato, M. C. R. (2005). Qualidade de vida e sintomas psicopatológicos do estudante universitário trabalhador (Dissertação de Mestrado não publicada). Pontifícia Universidade Católica de Campinas, Campinas.

Tran, A., Tran, L., Geghre, N., Darmon, D., Rampal, M., Brandone, D., Gozzo, J. M., Haas, H., Rebouillat-Savy, K., Caci, H., \& Avillach, P. (2017). Health assessment of french university students and risk factors associated with mental health disorders. PLoS One, 12(11), e0188187. 
Tricco, A. C., Lillie, E., Zarin, W., O’Brien, K. K., Colquhoun, H., Levac, D., Moher, D., Peters, M. D. J., Horsley, T., Weeks, L., Hempel, S., Akl, E. A., Chang, C., McGowan, J., Stewart, L., Hartling, L., Aldcroft, A., Wilson, M. G., Garritty, C., Lewin, S., Godfrey, C. M., Macdonald, M. T., Langlois, E. V., Soares-Weiser, K., Moriarty, J., Clifford, T., Tunçalp, Ö., \& Straus, S. E. (2018). PRISMA Extension for Scoping Reviews (PRISMA-ScR): checklist and Explanation. Annals of Internal Medicine, 169(7), 467-473. http://dx.doi.org/10.7326/M18-0850.

Vicente, A. L. C. (2014). La salud mental en los portugueses de enseñanza superior frente a la transición al mercado de trabajo. International Journal of Developmental and Educational Psychology - Revista INFAD de Psicologia, 4(1), 433-450.

Witt, P. A., Monareng, L., Abraham, A. A., Koor, S., \& Saber, R. (2019). Resilience in occupational therapy students. South African Journal of Occupational Therapy, 49(2), 33-41.

World Federation of Occupational Therapists - WFOT. (2016). Minimum standards for the education of occupational therapists: revised 2016. Recuperado em 18 de junho de 2020, de www.wfot.org/ResourceCentre

\section{Contribuiçáo dos Autores}

Teresinha Cid Constantinidis e Thelma Simóes Matsukura trabalharam igualmente na concepçáo, redação do texto, organização das fontes, análises e discussão dos resultados. Todos os autores aprovaram a versão final do texto.

\section{Autor para correspondência}

Teresinha Cid Constantinidis

e-mail: teracidc@gmail.com

\section{Editora de Seçáo}

Profa. Dra. Daniela Tavares Gontijo 